2010-01-01

\title{
Strain Sensor Based on a Pair of Single-Mode-Multimode-Single- Mode Fiber Structures in a Ratiometric Power Measurement Scheme
}

\author{
Agus Hatta \\ Technological University Dublin, ahatta@tudublin.ie \\ Yuliya Semenova \\ Technological University Dublin, yuliya.semenova@tudublin.ie \\ Qiang wu \\ Technological University Dublin, qiang.wu@tudublin.ie
}

See next page for additional authors

Follow this and additional works at: https://arrow.tudublin.ie/engscheceart

Part of the Electrical and Computer Engineering Commons

\section{Recommended Citation}

Hatta, A. et al. (2010) Strain Sensor Based on a Pair of Single-Mode-Multimode-Single-Mode Fiber Structures in a Ratiometric Power Measurement Scheme. Applied Optics. Vol.49, no.3. doi:10.1364/ A0.49.000536

This Article is brought to you for free and open access by the School of Electrical and Electronic Engineering at ARROW@TU Dublin. It has been accepted for inclusion in Articles by an authorized administrator of ARROW@TU Dublin. For more information, please contact arrow.admin@tudublin.ie, aisling.coyne@tudublin.ie, gerard.connolly@tudublin.ie.

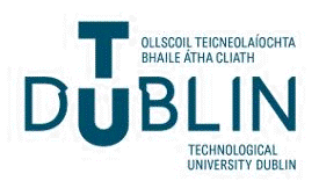




\section{Authors}

Agus Hatta, Yuliya Semenova, Qiang wu, and Gerald Farrell

This article is available at ARROW@TU Dublin: https://arrow.tudublin.ie/engscheceart/90 


\title{
Strain sensor based on a pair of single-mode- multimode-single-mode fiber structures in a ratiometric power measurement scheme
}

\author{
Agus Muhamad Hatta, ${ }^{1,2, *}$ Yuliya Semenova, ${ }^{1}$ Qiang $\mathrm{Wu},{ }^{1}$ and Gerald Farrell ${ }^{1}$ \\ ${ }^{1}$ Photonics Research Center, School of Electronic and Communications Engineering, \\ Dublin Institute of Technology, Kevin Street, Dublin 8, Ireland \\ ${ }^{2}$ On leave from Engineering Physics Department, Institut Teknologi Sepuluh Nopember (ITS), Surabaya, Indonesia \\ ${ }^{*}$ Corresponding author: ahatta@dit.ie
}

Received 29 October 2009; revised 17 December 2009; accepted 17 December 2009; posted 18 December 2009 (Doc. ID 119225); published 20 January 2010

\begin{abstract}
The strain and temperature dependencies of a step-index single-mode-multimode-single-mode (SMS) fiber structure are investigated numerically and experimentally. For intensity-based strain measurement using a single SMS fiber structure, at a selected wavelength, it is found that there is a high strain dependence, but also a temperature dependence that will induce strain measurement error. To minimize the temperature-induced strain measurement error, two SMS fiber structures are proposed and demonstrated in a ratiometric power measurement scheme; one SMS structure acts as the strain sensor, and the other SMS structure acts as the temperature monitor. The extracted temperature information is used to determine a strain value based on a suitable calibration of strain responses with temperature variations. It is demonstrated that for strain measurement from 0 to $1000 \mu \varepsilon$ within the temperature range from $10^{\circ} \mathrm{C}$ to $40^{\circ} \mathrm{C}$, the proposed configuration can provide a strain and temperature resolution of $0.34 \mu \varepsilon$ and $0.14^{\circ} \mathrm{C}$, respectively, with a temperature-induced strain measurement error as low as $0.39 \mu \varepsilon$. (C) 2010 Optical Society of America

OCIS codes: $\quad 060.2310,060.2370$.
\end{abstract}

\section{Introduction}

Fiber optic sensors are widely used for strain measurement in structural health monitoring. It is well known that temperature can induce a strain measurement error for fiber-optics-based strain sensors. Therefore, many techniques have been proposed to compensate for temperature dependence or to simultaneously measure strain and temperature, most commonly based on a fiber Bragg grating (FBG) [1-3]. In a FBG-based sensor, techniques employing an optical spectrum analyzer (OSA) are commonly used to extract wavelength information due to strain or temperature variation.

0003-6935/10/030536-06\$15.00/0

(C) 2010 Optical Society of America
Recently, a single-mode-multimode-single-mode (SMS) fiber structure has been investigated for various applications because of its low cost and ease of fabrication. Several applications of a SMS have been demonstrated, including a refractometer, filters, and temperature sensors [4-8]. The characteristics of the wavelength shift of the SMS spectral response due to strain and temperature applied to a step-index SMS fiber structure were investigated previously in [9]. In [10], a SMS fiber structure employing a graded index multimode fiber (MMF) was studied with regard to the sensing applications of wavelength shift due to strain and temperature. In [11], a SMS fiber structure combined with a FBG $\overline{\text { is }}$ utilized to enhance the sensitivity of strain measurement. A FBG combined with a SMS fiber structure has also been reported for simultaneous strain and temperature measurement [12]. However, all of these strain 
measurement techniques, with or without temperature determination, require the use of an OSA.

In addition to the disadvantage of needing an OSA, in a SMS fiber structure the wavelength shifts in the spectral response due to increases in strain or temperature are in opposite directions, shifting to a lower or higher wavelength, respectively, as in $[9,10]$. Therefore, when a single SMS fiber structure is used as a strain sensor where there are ambient temperature variations, accurate strain measurement can be carried out only when temperature information is known. In this paper, the use of a pair of SMS fiber structures is proposed and demonstrated as a strain sensor with self-temperature monitoring. Additionally, an intensity-based measurement scheme rather than an OSA is used. Two SMS fiber structures are used; one as a strain sensor and the other as a temperature monitor that is located in the same thermal environment. A ratiometric power measurement scheme is used that offers low cost, simple configuration, and the potential for high-speed measurement compared with the techniques that employ an OSA.

\section{Strain and Temperature Dependence of a Step- Index SMS Fiber Structure}

A SMS fiber structure as shown in Fig. 1(a) is formed by splicing a step-index MMF between two standard single-mode fibers (SMFs). Figure 1(b) shows the schematic of a system utilizing a pair of SMS fiber structures for strain sensor with self-temperature monitoring in a ratiometric power measurement technique. The input signal is divided into two paths and connected to a pair of SMS fiber structures and a single reference arm. Both SMS fiber structures, SMS-1 and SMS-2, are located together and therefore experience the same ambient temperature. The strain is only applied to the SMS-1. At a chosen fixed input wavelength, strain applied to SMS-1 changes the output power ratio $\mathrm{R} 1$, where $\mathrm{R} 1=\mathrm{P} 1$ Pref in decibels. The output power ratio $\mathrm{R} 1$ variation tracks the variation in strain. SMS-2 is used as a temperature monitor, so that the variation in the output power ratio $\mathrm{R} 2$, where $\mathrm{R} 2=\mathrm{P} 2$ - Pref in deci-

(a)
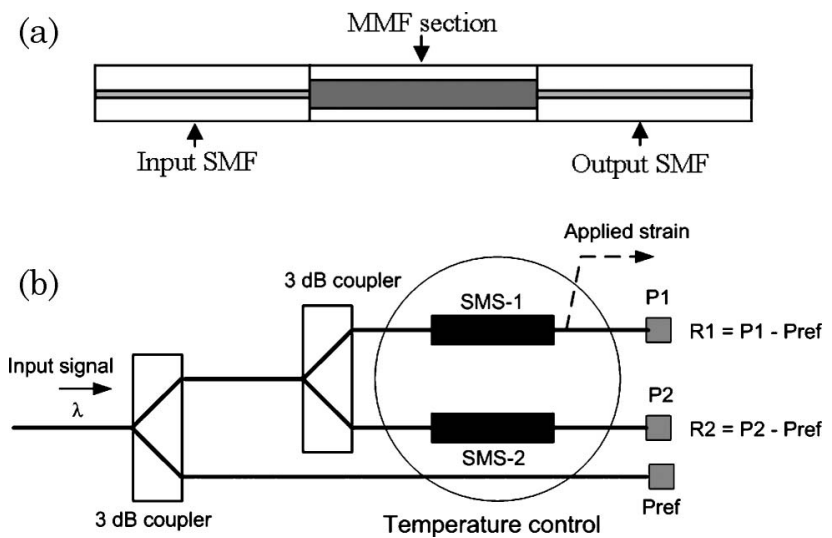

Fig. 1. (a) Single SMS fiber structure (b) Schematic structure of strain measurement with a self-temperature monitoring in a ratiometric power measurement scheme using a pair of SMS fibers. bels, tracks temperature variations. However, the presence of a temperature fluctuation also affects SMS-1 and changes the output power P1 and thus R1. To determine the applied strain accurately with ambient temperature variations, the strain independent temperature information obtained from R2 is used to accurately extract the value of applied strain on its own, even in the presence of temperatureinduced variations in $\mathrm{R} 1$. This assumes that a suitable calibration of $\mathrm{R} 1$ to account for the effect of strain and temperature has taken place.

To optimize a SMS fiber structure for the application described here and to select a suitable operating wavelength, it is useful to initially investigate the strain and temperature dependence of the spectral response of a single SMS fiber structure as in Fig. 1(a). A modal propagation analysis for linearly polarized modes can be used to investigate light propagation in the SMS fiber structure [13]. It is known that an applied strain $(\varepsilon)$ and a temperature variation $(\Delta T)$ induce a change in the length $(\Delta L)$ and the core radius $(\Delta a)$ of the step-index MMF section of the SMS fiber structure as well as a change in the refractive index $(\Delta n)$ of the SMF and MMF sections. For an applied strain $(\varepsilon)$, the change in MMF length, core radius, and refractive index can be expressed as $[\underline{9}]$

$$
\Delta L=L \varepsilon,
$$

$$
\Delta a_{(\mathrm{SMF}, \mathrm{MMF})}=-\sigma a_{(\mathrm{SMF}, \mathrm{MMF})} \varepsilon
$$

$$
\begin{aligned}
\Delta n_{(\mathrm{SMF}, \mathrm{MMF}) i} & =-\frac{n_{(\mathrm{SMF}, \mathrm{MMF})_{i}{ }^{3}}}{2}\left[p_{12}-\sigma\left(p_{11}+p_{12}\right)\right] \varepsilon \\
& =-p_{e} \varepsilon
\end{aligned}
$$

where $L$ is the length of the MMF section, $a$ is the core radius, $n_{i}$ represents the refractive indices of the core and cladding of the MMF, $p_{11}$ and $p_{12}$ are the strainoptic coefficients for fused silica, $p_{e}$ is the effective strain-optic coefficient, and $\sigma$ is the Poisson ratio. The change in length for the MMF, core radius, and refractive index due to the temperature can be expressed as [9]

$$
\Delta L=\alpha L \Delta T
$$

$$
\Delta a_{(\mathrm{SMF}, \mathrm{MMF})}=\alpha a_{(\mathrm{SMF}, \mathrm{MMF})} \Delta T,
$$

$$
\Delta n_{(\mathrm{SMF}, \mathrm{MMF}) i}=\beta n_{(\mathrm{SMF}, \mathrm{MMF}) i} \Delta T
$$

where $\alpha$ is a thermal expansion coefficient and $\beta$ is a thermo-optic coefficient. The combined effect of strain and temperature on the length of the MMF, its core radius, and the refractive index are 


$$
\Delta L=L \varepsilon+\alpha L \Delta T
$$

$$
\begin{aligned}
\Delta a_{(\mathrm{SMF}, \mathrm{MMF})}= & -\sigma a_{(\mathrm{SMF}, \mathrm{MMF})} \varepsilon \\
& +\alpha a_{(\mathrm{SMF}, \mathrm{MMF})} \Delta T,
\end{aligned}
$$

$$
\Delta n_{(\mathrm{SMF}, \mathrm{MMF}) i}=-p_{e} \varepsilon+\beta n_{(\mathrm{SMF}, \mathrm{MMF}) i} \Delta T .
$$

In this paper, the core/cladding diameter of the SMF is $9 / 125 \mu \mathrm{m}$ and of the MMF is $105 / 125 \mu \mathrm{m}$. It is known that MMF lengths from 44.53 to $42.45 \mathrm{~mm}$ provide a bandpass spectral response with a peak bandpass wavelength that depends on the length, ranging from 1490 to $1560 \mathrm{~nm}$, respectively [13]. In this investigation a MMF length of $44.38 \mathrm{~mm}$ is chosen to provide an operating wavelength for the sensor in the region of $1490-1560 \mathrm{~nm}$. The spectral response of the SMS fiber structure is calculated by using modal propagation analysis and is shown in the inset in Fig. 2. To determine which wavelength is most sensitive to strain and temperature, the strain-dependent loss (SDL) and temperature-dependent loss (TDL) are calculated from 1490 to $1560 \mathrm{~nm}$. The SDL is calculated at $\Delta \varepsilon=1000 \mu \varepsilon$ and the TDL at $\Delta T=30^{\circ} \mathrm{C}$. The strain- and temperature-related coefficient values $p_{e}=0.22, \quad \sigma=0.16, \quad \alpha=5 \times$ $10^{-7} /{ }^{\circ} \mathrm{C}$ and $\beta=6.9 \times 10^{-6} /{ }^{\circ} \mathrm{C}$ are used as in [9]. Figure 2 shows the calculated SDL and TDL of the SMS fiber structure, from which it is clear that some wavelengths are much more sensitive to strain and temperature, especially at the dip (1535-1545 nm) region of the bandpass response.

Given that a fixed wavelength ratiometric power measurement system is used for interrogation, the transmission losses responses due to strain and temperature are calculated at an operating wavelength of $1539 \mathrm{~nm}$. This wavelength is chosen because of its high SDL value, which will improve strain sensitivity. In Fig. 3(a), the transmission loss responses due to an applied strain from 0 to $1000 \mu \varepsilon$ at $T=0,10,20$,

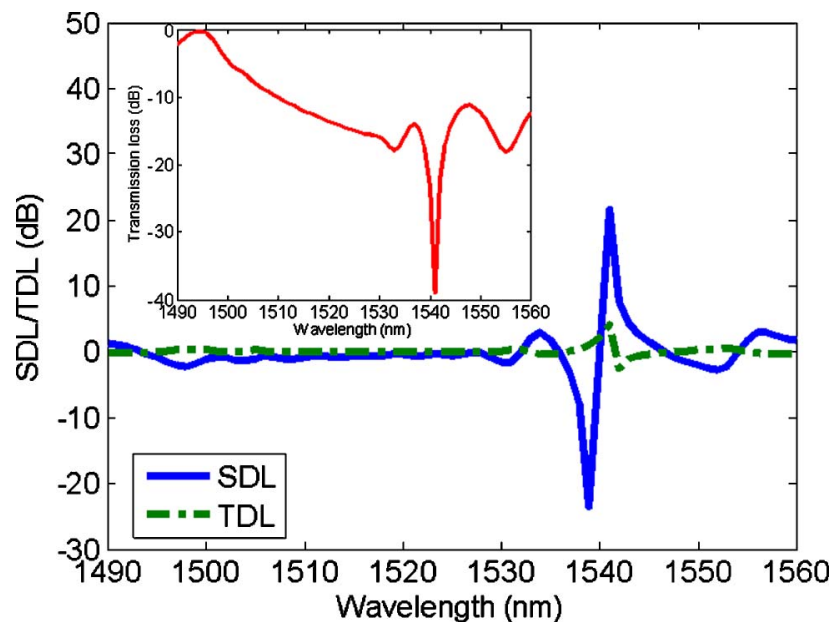

Fig. 2. (Color online) SDL and TDL of the SMS fiber structure. Inset, spectral response.
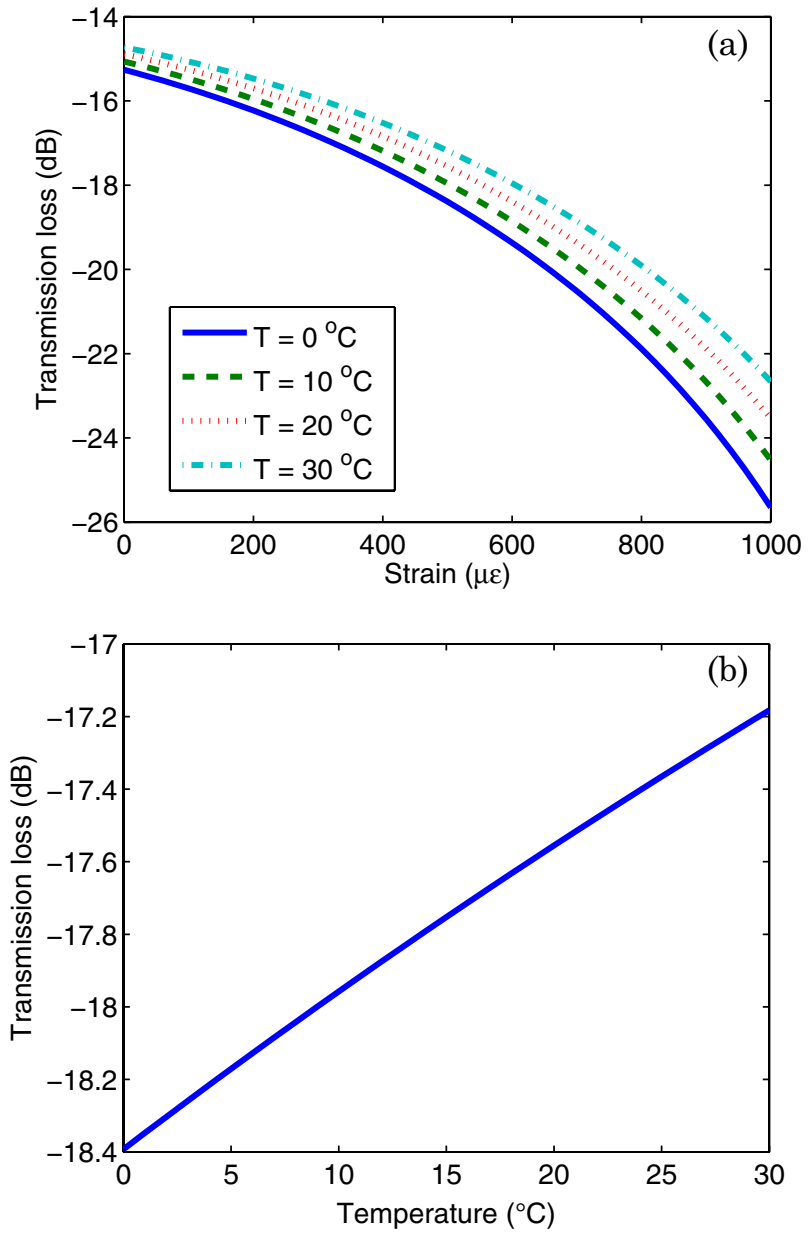

Fig. 3. (Color online) Transmission loss responses at an the operating wavelength of $1539 \mathrm{~nm}$ : (a) strain responses at several ambient temperatures, (b) temperature response for an applied strain of $500 \mu \varepsilon$.

$30^{\circ} \mathrm{C}$ are shown. It is clear that the response to strain is monotonically decreasing but that the presence of a temperature variation changes the transmission loss response to strain. At $T=0^{\circ} \mathrm{C}$, the discrimination range is $-10.389 \mathrm{~dB}$ which is equivalent to a strain sensitivity of $10.389 \times 10^{-3} \mathrm{~dB} / \mu \varepsilon$. To further illustrate the effect of temperature, the transmission loss response at $500 \mu \varepsilon$ due to a temperature variation from $0^{\circ} \mathrm{C}$ to $30^{\circ} \mathrm{C}$ is shown in Fig. 3(b). A temperature change of $30^{\circ} \mathrm{C}$ alters the transmission loss response by $1.21 \mathrm{~dB}$ at $500 \mu \varepsilon$. Such a change in the transmission loss due to temperature is equivalent to a strain measurement error of $116.4 \mu \varepsilon$. It is clear from this result that the magnitude of transmission loss change, due to variations in temperature, is substantial and of the same order of magnitude as that due to the strain itself. Ambient temperature variations can thus significantly reduce the strain measurement accuracy if only one SMS fiber structure is used.

A SMS fiber structure when used as a strain sensor is clearly temperature sensitive. However, it is apparent from Fig. 3(a) that the transmission loss 
monotonically decreases for a decrease in temperature. This means that, by monitoring the system temperature, it is feasible to apply a calibration correction factor to minimize the temperature-induced strain measurement error. Furthermore, the monotonically decreasing transmission loss response with temperature means that, by using two identical SMS fiber structures, one as the strain sensor and the other as a temperature monitor, a strain sensor with self-temperature monitoring can be implemented as presented in Fig. 1(b).

\section{Experimental Results}

The SMS fiber structure described above was fabricated by using a precision Fujikura CT-07 cleaver and a Sumitomo type-36 three-axis fusion splicer. Two such SMS fiber structures were fabricated with a MMF length of $44.38 \mathrm{~mm}$. The lengths of the two MMFs were carefully controlled and cleaved in order to minimize length differences. The transmission loss responses of the fabricated SMS fiber structures were measured by using a tunable laser TUNIC PLUS and a powermeter as shown in Fig. 4. The measured results show a good agreement with the calculated result by comparison with the inset in Fig. 2 . The measured results for the spectral responses of the two fabricated SMS structures are most identical. The small discrepancy between two measured spectral responses is most likely caused by residual MMF length errors and also fiber core splice offsets, occurring during the fabrication process [14].

The two SMS fiber structures are utilized in a ratiometric measurement scheme as shown in Fig. 1(b). The input signal from the tunable laser is split into two equal-intensity signals by using a $3 \mathrm{~dB}$ fiber coupler. One of the signals passes through the reference arm, while the other passes via a second $3 \mathrm{~dB}$ fiber coupler to arms containing the pair of SMS fiber structures SMS-1 and SMS-2. Powermeters are placed at the ends of all three arms. Axial strain is applied to SMS-1, and both SMS fiber structures are attached to the same thermoelectric Peltier

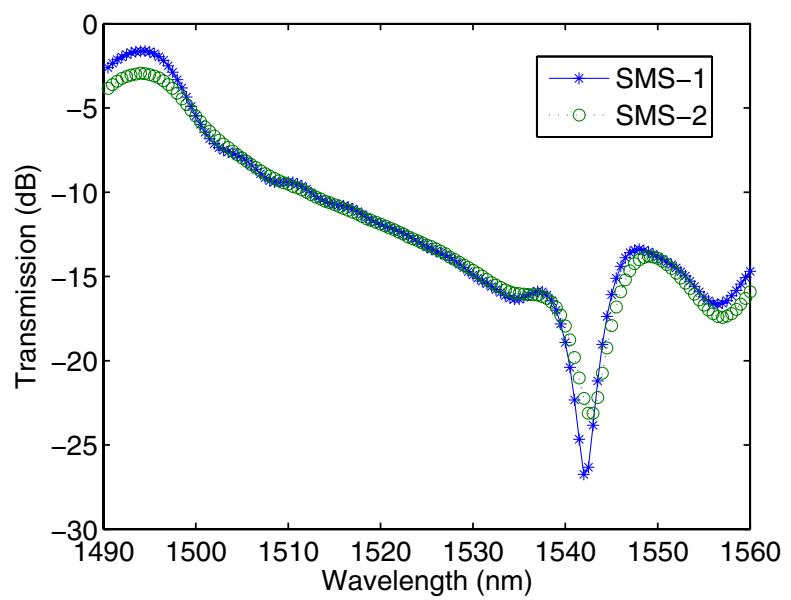

Fig. 4. (Color online) Measured spectral response of two SMS fiber structures. cooler. At the selected wavelength of $1539 \mathrm{~nm}$, the ratio response due to the applied strain was measured from 0 to $1000 \mu \varepsilon$ with an increment of $100 \mu \varepsilon$ at the temperatures of $10^{\circ} \mathrm{C}, 20^{\circ} \mathrm{C}, 30^{\circ} \mathrm{C}$, and $40^{\circ} \mathrm{C}$. The measured ratio $R 1$, as defined in Fig. $1(b)$, is presented in Fig. 5(a). Figure 5(b) shows the calculated ratio responses from 0 to $\overline{1000} \mu \varepsilon$ at temperature of $10^{\circ} \mathrm{C}, 20^{\circ} \mathrm{C}, 30^{\circ} \mathrm{C}$, and $40^{\circ} \mathrm{C}$, taking account of a $3 \mathrm{~dB}$ coupler loss. The measured strain sensitivity at $10^{\circ} \mathrm{C}$ is $8.732 \times 10^{-3} \mathrm{~dB} / \mu \varepsilon$, which is approximately equal to the calculated strain sensitivity of $9.463 \times$ $10^{-3} \mathrm{~dB} / \mu \varepsilon$ from Fig. 5(b). The measured and calculated ratio responses for strain with temperature variations also show good agreement. Discrepancies can be attributed to splicing loss during fabrication and the accuracy of the strain and temperature coefficient values used in the calculations.

In the previous section it was found that temperature can significantly degrade the strain measurement accuracy of a single SMS fiber structure. It is shown in Fig. 5(a) that the ratio variation due to a temperature change from $10^{\circ} \mathrm{C}$ to $40^{\circ} \mathrm{C}$ is $0.736 \mathrm{~dB}$ at $500 \mu \varepsilon$. This ratio variation induces a strain measurement error of $84.3 \mu \varepsilon$.
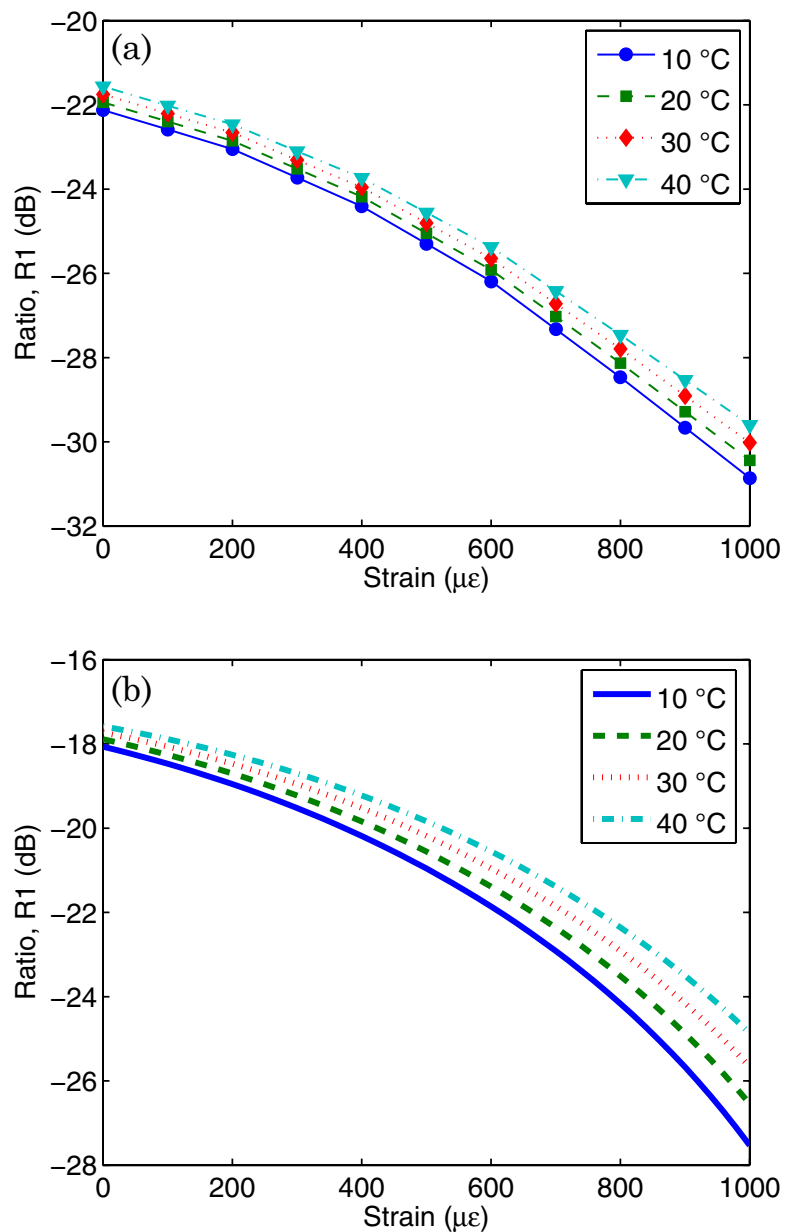

Fig. 5. (Color online) Ratio response of SMS-1 as a function of strain with temperature variation at an operating wavelength of $1539 \mathrm{~nm}$ : (a) measured, (b) calculated. 
To minimize the temperature-induced strain measurement error, self-temperature monitoring can be carried out by employing SMS-2. The measured and calculated ratio responses due to a temperature variation from $10^{\circ} \mathrm{C}$ to $40^{\circ} \mathrm{C}$ are shown in Fig. 6(a) and $6(\mathrm{~b})$, respectively. The ratio response is monotonically increasing, with an acceptable measured discrimination range of $0.623 \mathrm{~dB}$ (calculated value is $0.533 \mathrm{~dB}$ ), which is suitable for the temperature monitoring. Discrepancies between the measured and the calculated values can be attributed to insertion losses, such as splice loss, the accuracy of the strain and temperature coefficient values used in the calculations, and, most important, residual MMF length errors.

To analyze the effect of residual MMF length errors on the temperature response, Fig. 7 shows the calculated ratio responses due to temperature variation from $10^{\circ} \mathrm{C}$ to $40^{\circ} \mathrm{C}$ with $\mathrm{MMF}$ length errors of $\Delta L_{\mathrm{MMF}}=-20,0,20$, and $40 \mu \mathrm{m}$. It is clear that both the insertion loss and the discrimination range of the calculated ratio response are influenced by length errors in the MMF section. The discrimination ranges are $0.195,0.533,0.995$, and $1.792 \mathrm{~dB}$, respectively.
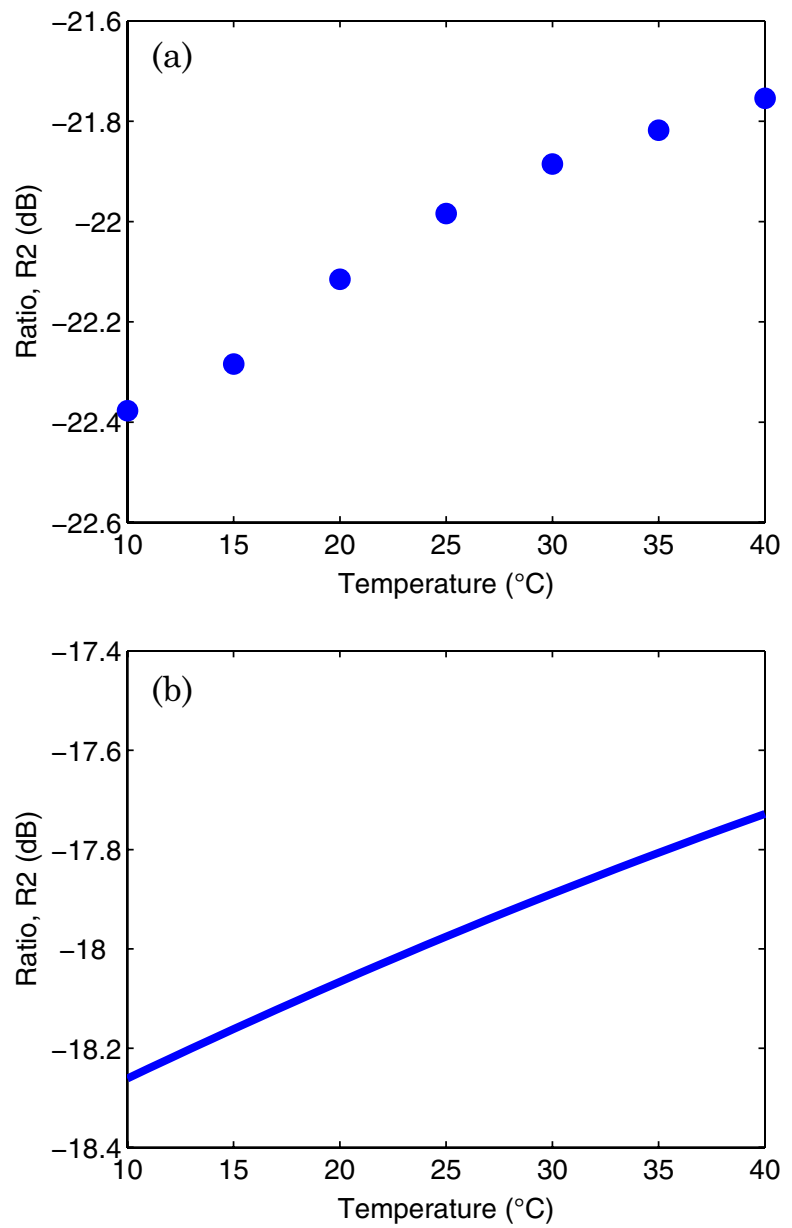

Fig. 6. (Color online) Ratio response of SMS-2 due to temperature variation at an operating wavelength of $1539 \mathrm{~nm}$ : (a) measured, (b) calculated.
The linearity of the calculated ratio response also depends on the length of the MMF. The calculated linear regression correlation coefficients for the various length errors in Fig. $\underline{7}$ are 0.993, 0.998, 0.999, and 0.996 , respectively. By comparison the linear regression correlation coefficient of the measured ratio response is 0.975 as in Fig. 6(a).

From the measured results as in Fig. $\underline{5}$, it is estimated that a strain measurement resolution of $0.34 \mu \varepsilon$ is possible, assuming the uncertainty in the measured power ratio is $0.003 \mathrm{~dB}$ [15]. From Fig. 6, the temperature measurement resolution is estimated as $0.14^{\circ} \mathrm{C}$. From the extracted temperature information, an accurate strain value can be determined based on a suitable calibration of strain responses with temperature variations. The temperature-induced strain measurement error at $500 \mu \varepsilon$ can be reduced from $84.3 \mu \varepsilon$ to as low as $0.39 \mu \varepsilon$.

For a given fixed signal source wavelength, the length of the MMF sections of SMS-1 and SMS-2 will need to be optimized to achieve the highest strain and temperature dependence, respectively. It is shown in [13] that the peak wavelength of a bandpass filter can be tuned by selecting a suitable MMF length. It should be noted that, to achieve high sensitivity for strain measurement with low temperature-induced errors with a fixed wavelength source, the MMF lengths of SMS-1 and SMS-2 need to be optimized in such a way that the edge of a dip of the SMS transmission spectrum coincides exactly with the operating wavelength.

In a ratiometric power measurement, the influence of the signal-to-noise ratio of the laser source can degrade the measurement resolution [16], as well as the sensitivity of the sensor itself. According to [16], measurement resolution can be maintained if the signal-to-noise ratio of the laser source is higher than $40 \mathrm{~dB}$ for a typical sensor response with a minimum ratio response of $-35 \mathrm{~dB}$. In this ratiometric system, the signal-to-noise ratio of the laser source used is $55 \mathrm{~dB}$, and the minimum measured ratio response of $\mathrm{R} 1$ and $\mathrm{R} 2$ is higher than $-35 \mathrm{~dB}$, as in

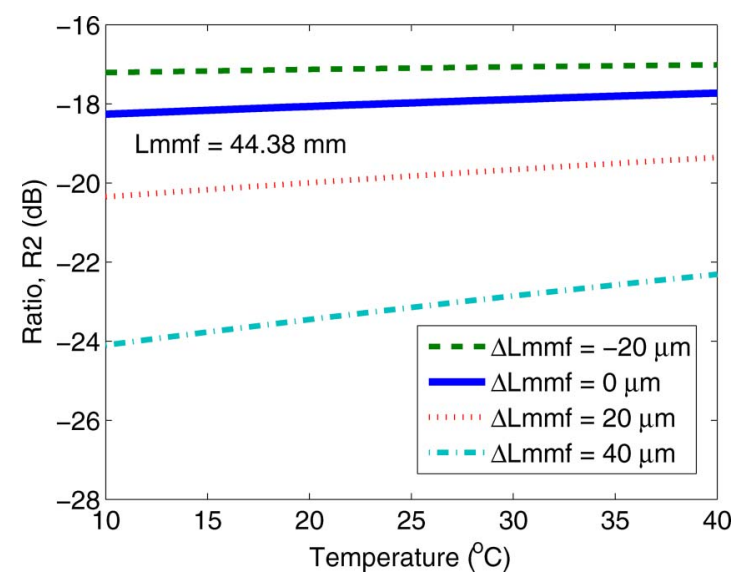

Fig. 7. (Color online) Calculated ratio response of SMS-2 with MMF length errors due to temperature variation at an operating wavelength of $1539 \mathrm{~nm}$. 
Fig. 5(a) and 6(a), to maintain the measurement resolution.

\section{Conclusions}

The strain and temperature dependence of an SMS fiber structure has been investigated numerically and experimentally. It is found that a single SMS fiber structure for an intensity-based measurement strain sensor exhibits substantial strain error measurement due to temperature variations. It has also been demonstrated that strain measurement using a pair of SMS fiber structures within a ratiometric power measurement scheme can minimize temperature-induced strain measurement error. In the proposed configuration, SMS-1 acts as a strain sensor and SMS-2 acts as a temperature sensor. At an operating wavelength of $1539 \mathrm{~nm}$, for strain measurement from 0 to $1000 \mu \varepsilon$ within a temperature range from $10^{\circ} \mathrm{C}$ to $40^{\circ} \mathrm{C}$, SMS-1 can provide strain measurement resolution of $0.34 \mu \varepsilon$ but with a temperature-induced strain measurement error of $84.3 \mu \varepsilon$ at $500 \mu \varepsilon$. The SMS-2 can provide temperature measurement resolution of $0.14^{\circ} \mathrm{C}$. Utilizing SMS-1 and SMS-2 in the proposed configuration, the temperature-induced strain measurement error can be reduced significantly to $0.39 \mu \varepsilon$.

\section{References}

1. G. P. Brady, K. Kalli, D. J. Webb, D. A. Jackson, L. Reekie, and J. L. Archambault, "Simultaneous measurement of strain and temperature using the first- and second-order diffraction wavelengths of Bragg gratings," IEE Proc. Optoelectron. 144(3), 156-161 (1997).

2. F. M. Hanran, J. K. Rew, and P. D. Foote, "A strain-isolated fibre Bragg grating sensor for temperature compensation of fibre Bragg grating strain sensors," Meas. Sci. Technol. 9, pp. 1163-1166 (1998).

3. L. V. Nguyen, D. Hwang, D. S. Moon, and Y. Chung, "Simultaneous measurement of temperature and strain using a Lyot fiber filter incorporated with a fiber Bragg grating in a linear configuration," Meas. Sci. Technol. 20, 034006 (2009).
4. Q. Wang and G. Farrell, "All-fiber multimode-interference based refractometer sensor: proposal and design," Opt. Lett. 31, 317-319 (2006).

5. W. S. Mohammed, P. W. E. Smith, and X. Gu, "All-fiber multimode interference bandpass filter," Opt. Lett. 31, 2547-2549 (2006).

6. A. M. Hatta, G. Farrell, Q. Wang, G. Rajan, P. Wang, and Y. Semenova, "Ratiometric wavelength monitor based on singlemode-multimode-singlemode fiber structure," Microwave Opt. Technol. Lett. 50, 3036-3039 (2008).

7. E. Li and G.-D. Peng, "Wavelength-encoded fiber-optic temperature sensor with ultra-high sensitivity," Opt. Commun. 281, 5768-5770 (2008).

8. A. M. Hatta, G. Rajan, Y. Semenova, and G. Farrell, "A SMS fiber structure for temperature measurement using a simple intensity based interrogation system," Electron. Lett. 45, 1069-1071 (2009).

9. E. Li, "Temperature compensation of multimode-interference based fiber devices," Opt. Lett. 32, 2064-2066 (2007).

10. S. M. Tripathi, A. Kumar, R. K. Varshney, Y. B. P. Kumar, E. Marin, and J. P. Meunier, "Strain and temperature sensing characteristics of single-mode-multimode-singlemode structures," J. Lightwave Technol. 27, 2348-2356 (2009).

11. E. Li, "Sensitivity-enhanced fiber-optic strain sensor based on interference of higher order modes in circular fibers," IEEE Photon. Technol. Lett. 19, 1266-1268 (2007).

12. D. P. Zhou, L. Wei, W. K. Liu, Y. Liu, and J. W. Y. Lit, "Simultaneous measurement for strain and temperature using fiber Bragg gratings and multimode fibers," Appl. Opt. 47, 16681672 (2008).

13. Q. Wang, G. Farrell, and W. Yan, "Investigation on singlemodemultimode-singlemode fiber structure," J. Lightwave Technol. 26, 512-519 (2008).

14. A. M. Hatta, G. Farrell, P. Wang, G. Rajan, and Y. Semenova, "Misalignment limits for a singlemode-multimode-singlemode fiber based edge filter," J. Lightwave Technol. 27, 2482-2488 (2009).

15. Q. Wang, G. Rajan, P. Wang, and G. Farrell, "Resolution investigation of a ratiometric wavelength measurement system," Appl. Opt. 46, 6362-6367 (2007).

16. Q. Wang, G. Farrell, and T. Freir, "Study of transmission response of edge filters employed in wavelength measurements," Appl. Opt. 44, 7789-7792 (2005). 